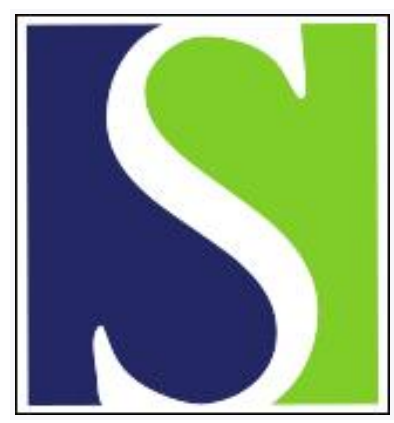

Scand J Work Environ Health 2002;28(6):371-385

https://doi.org/10.5271/sjweh.689

Issue date: Dec 2002

Dermal exposure assessment in occupational epidemiologic research

by Vermeulen $\mathrm{R}$, Stewart $\mathrm{P}$, Kromhout $\mathrm{H}$

Affiliation: Occupational Epidemiology Branch, Division of Cancer Epidemiology and Genetics, National Cancer Institute, 6120 Executive Boulevard, Rockville, MD 20892, Unites States. vermeulr@mail.nih.gov

Refers to the following texts of the Journal: 1995;21(2):124-133 1998;24 suppl 2:42-53 1998;24 suppl 2:25-41 1995;21(1):30-35 1996;22(2):94-101 1996;22(6):425-432

The following articles refer to this text: 2002;28(6):367-369;

2006;32(3):209-218

Key terms: dermal exposure assessment; dermal uptake; epidemiology; exposure assessment; exposure variability; occupational epidemiologic research; review; skin exposure

This article in PubMed: www.ncbi.nlm.nih.gov/pubmed/12539797 


\title{
Dermal exposure assessment in occupational epidemiologic research
}

\author{
by Roel Vermeulen, PhD, ${ }^{1,2}$ Patricia Stewart, PhD, ${ }^{1}$ Hans Kromhout, PhD²
}

\begin{abstract}
Vermeulen R, Stewart P, Kromhout H. Dermal exposure assessment in occupational epidemiologic research. Scand J Work Environ Health 2002;28(6):371-385.

Recognition of the importance of skin exposure in industrial settings has steadily increased over the last few decades. Unfortunately, the growing attention to dermal exposure in industrial hygiene has often not been reflected in the field of occupational epidemiology. An extensive literature survey was conducted to identify dermal exposure assessment methods that have been applied in epidemiologic studies. Subsequently, methodologies are postulated that could be applied to epidemiologic research. Attention is given to intensity, frequency, and duration of exposure, the exposed surface area, and personal, temporal and spatial variability in dermal exposure and uptake. It is anticipated that, in the near future, dermal exposure assessment in epidemiologic research will be based generally on expert judgment and to some degree on process-specific exposure models. Field studies collecting quantitative dermal exposure data and statistical modeling to identify exposure determinants will, however, be imperative if progress is to be made in the field of dermal exposure assessment for epidemiologic purposes.
\end{abstract}

Key terms epidemiology, exposure assessment, exposure variability, dermal uptake, review, skin exposure.

Recognition of the importance of skin exposure in industrial and environmental settings has steadily increased over the last few decades. Whereas the 1980s saw widespread recognition of the importance of skin exposure in the field of industrial hygiene, the 1990s saw the beginnings of a systematic characterization of the field $(1,2)$. Unfortunately, the growing attention to dermal exposure has rarely been reflected in the field of occupational epidemiology. Although it is widely recognized that exposure to hazardous substances may occur by inhalation, ingestion, and dermal contact, the focus of exposure assessment in occupational epidemiology traditionally, explicitly, or implicitly has been on inhalation exposures. This situation is even true for contaminants, for example, pesticides, polycyclic aromatic hydrocarbons, and polychlorinated biphenyls (PCB), and solvents and diseases such as dermatitis and skin cancer, for which dermal exposure is known to contribute significantly to internal dose or the disease.
Valid and reliable exposure assessments are crucial in occupational epidemiology because new risks are likely to be lower than those seen historically and they are therefore more difficult to detect. They are also crucial because the emphasis in epidemiology has shifted from qualitative risk identification to quantitative risk assessment, which incorporates exposure-response relationships (3). Thus it is important that exposures through multiple routes (ie, oral, dermal, and inhalation) and from different sources (ie, occupational, environmental, and dietary) be accurately assessed in epidemiologic research. Inaccurate and imprecise exposure estimates may lead to a loss of power, precision, and attenuation in health risk estimates, depending on the type of error structure $(4,5)$.

Proper exposure assessment strategies for estimating dermal exposure in epidemiologic research depend on the chosen study design, for example, prospective, crosssectional, retrospective (cohort, case-referent), and the

1 Occupational Epidemiology Branch, Division of Cancer Epidemiology and Genetics, National Cancer Institute, Rockville, Maryland, United States.

2 Institute of Risk Assessment Sciences, Environmental and Occupational Health Group, Utrecht University, Utrecht, The Netherlands.

Correspondence to: Dr Roel Vermeulen, Occupational Epidemiology Branch, Division of Cancer Epidemiology and Genetics, National Cancer Institute, 6120 Executive Boulevard, Rockville, MD 20892, USA. [E-mail: vermeulr@mail.nih.gov] 
health outcome of interest (eg, chronic, acute, systemic, local). In prospective and cross-sectional studies, one has the opportunity to collect current dermal exposure data and information on possible determinants that could subsequently be used to estimate group or individual dermal exposure levels. However, in retrospective studies one depends on historical exposure information or expert judgment. The health outcome under consideration determines the locations where measurements should be made. In the case of local effects like hand dermatitis, dermal exposure is important at the body location of interest, while for systemic effects the total dermal body exposure is the key for estimating internal dose.

We conducted a literature survey to identify dermal exposure assessment methods that have been applied in epidemiologic research. Using the identified studies and recognized determinants of dermal exposure and uptake as a basis, we describe factors that should be considered and postulate methodologies that could be applied for dermal exposure assessment in occupational epidemiologic research. Attention is given to estimating the intensity and duration of exposure, the exposed surface area, personal, temporal and spatial variability in dermal exposure, and uptake. The focus of this paper is primarily on historical dermal exposure assessment in relation to systemic effects, but many of the considerations described apply to other study designs and local effects as well.

\section{Exposure metrics and analyses}

The dose surrogates commonly used in occupational epidemiology are exposure intensity, exposure duration, cumulative exposure, and average exposure over the work history. For chronic health outcomes cumulative exposure is generally thought to be the most appropriate measure of exposure (6). The dose surrogate most preferred, however, would be an estimate of the cumulative dose of the active substance or metabolite at the target organ. Biomarkers of exposure potentially reflect internal dose and have the advantage that they integrate exposure from all sources through all routes of exposure, including the dermal route. However, for most chemical exposures, no biomarkers of the (historical) internal dose are readily available, and therefore their current use is limited in (retrospective) exposure assessment. As a result, substances absorbed dermally will require, at least for the time being, the development of separate exposure estimates for both inhalation and dermal exposure.

Separate estimates of inhalation and dermal exposure in an epidemiologic study can be used in several ways. One could be to combine them into a single estimate of internal dose. This procedure would require, however, quantitative estimates of the two exposure routes in the same measurement units and information on the respiratory and dermal absorption rates. For most substances, such information is not readily available. Moreover, it is at least questionable whether accurate quantitative estimates can be derived for the dermal route with current measurement methods. [For an overview of dermal exposure assessment techniques see Brouwer et al (7), Cherrie et al (8), and Soutar et al (9).] Therefore, an integrated estimate of the internal dose cannot, in many cases, be derived easily.

The alternative is to develop separate estimates for inhalation and dermal exposure and use them as independent estimates of exposure or use them in a stratified analysis. This approach allows the simultaneous use of quantitative inhalation estimates and semiquantitative or qualitative dermal estimates in the same analysis. The stratified approach does, however, have consequences for the statistical power of the study, and its applicability is, in many studies, limited to exposures with a moderate-to-high prevalence.

\section{Assessment of dermal exposure in occupational epidemiologic studies}

Historically, several different exposure indices have been used for inhalation exposure in occupational epidemiologic research, varying from simple surrogates of exposure to sophisticated measures of internal dose (3, 10 ) and from qualitative (exposed, unexposed) and semiquantitative (low, medium, high) estimates to quantitative measures. Because the latency of most chronic diseases is now within the time frame in which historical airborne measurements are available, deterministic and stochastic modeling of historical inhalation exposure data has become possible and therefore enables an evaluation of quantitative exposure-response relationships (11-13).

Unfortunately, the evaluation of dermal exposure is less developed. This lack of development is, in part, due to the scarceness of quantitative exposure data, and standardized methods for quantifying dermal exposure to many substances are still lacking (14). Only a limited number of epidemiologic studies can be found in the peer-reviewed literature that has assessed dermal exposure in any way. It is, however, difficult to present a complete overview of the methods and indices used because much of the published literature does not explicitly indicate which exposure routes were considered in the exposure assessment. In fact, even when dermal 
exposure is identified as being considered, it is usually not clear how it was evaluated.

\section{Qualitative estimates}

Several qualitative estimates have been applied for dermal exposure in epidemiologic research. These estimates have almost exclusively been based on assumed determinants (subjective or objective) of dermal exposure, which were subsequently used as qualitative exposure proxies (yes;no). For example, in an epidemiologic study on acute pyrethroid poisoning among cotton farmers, dermal exposure was measured, and exposure determinants were identified (15). These determinants, contamination of clothing (yes;no), concentration of pyrethroid in the application solution (\%), and the occurrence of leakage or blockage of the sprayer (yes;no), were used in the epidemiologic analyses, and each was found to be related to neurological symptoms. A similar approach was used in a study of signs and symptoms of pesticide toxicity among Indonesian farmers (16). In this study each spray operation was observed, and variables thought to influence exposure were recorded. The following four types of variables were consequently considered: the frequency and mechanics of the spray operations (applications/week, mixing with bare hands, leaky equipment, spraying against the wind), contact of the clothing and body with the spray solution (percentage of spray operations, hands or feet becoming wet during mixing or pouring, splashed body during application), the type of clothing worn (footgear, gloves, eyeglasses, long pants, short-sleeved shirt, headgear), and the kind of chemicals sprayed and their management (pesticides, hazard grade of the World Health Organization, other chemicals). The number of applications per week, the use of hazardous pesticides (grade 1B and II, established by the World Health Organization), and skin and clothing becoming wet with the spray solution were found to be associated with neurobehavioral signs and symptoms.

\section{Semiquantitative estimates}

Semiquantitative estimates of dermal exposure were used in a study of adverse health effects among tannery workers $(17,18)$. The assessment of skin exposure was based on a three-point scale of no contact with the agent of interest, moderate contact (infrequent skin contact with the agent, eg, contact occurred during specific activities that were not part of the daily work routine), and frequent contact (frequent skin contact, eg, regular contact was unavoidable due to the activities performed daily). In a study of workers at a coal liquefaction plant, a similar ranking system was used, based primarily on the likelihood of skin contact with various products as assessed by expert judgment (minimal, low, medium, high) (19). Self-reported frequency of direct dermal contact with PCB was used in a study of clinical and metabolic abnormalities to divide PCB-exposed workers into four exposure groups (eg, never, rarely, occasionally, and frequent contact) (20). In a study of cancer mortality among workers exposed to acrylonitrile, a dermal score was developed by multiplying the percentage concentration of acrylonitrile in the liquid with the estimated frequency of dermal contact $(21,22)$.

\section{Quantitative estimates}

Only a handful of examples of studies developing quantitative estimates of dermal exposure were found in the literature. Brouwer et al (23) developed an algorithm for calculating a cumulative exposure index (milligrams/ lifetime) in a study of the health effects of pesticides in the flower-bulb industry in The Netherlands. The algorithm included the application method (tractor- or boom-, backpack-, bike-spraying), the method of mixing and loading (direct tank filling, pouring, or scooping), the method of bulb disinfection (manual or mechanical dipping), the application rate (milligrams per hectare), the bulb acreage (hectares), the number of applications, the number of bulbs disinfected per year (number of containers), and a protection factor for personal protective equipment (range: $0=$ no protection to $1=$ complete protection) (24). In a study of Pliofilm ${ }^{\mathrm{TM}}$ workers, exposure to benzene was calculated on the basis of the concentration of benzene in the cement being used, the number of skin contacts with the cement per day, the surface area of the contacted skin (square centimeters), and the contact time (hours per day) (25-27). Dermal uptake (milligrams per kilogram per day) was subsequently calculated using these dermal exposure estimates, the dermal absorption rate (milligram per square centimeter per hour), and body weight of the individual (kilograms). The absorbed dose was then converted to an estimate of the corresponding airborne concentration in parts per million based on the respiratory absorption rate of benzene.

\section{Basic parameters of dermal exposure}

The qualitative and quantitative parameters used in the occupational exposure algorithms across studies are remarkably similar and rely on or reflect the following three basic estimates: (i) the concentration or mass of the contaminant (eg, intensity), (ii) the exposed surface area, and (iii) the duration or frequency of the exposure. Not surprisingly, these basic parameters have also been 
described in several conceptual models for the assessment of dermal exposure $(1,28)$. In the following paragraphs these parameters are discussed in somewhat more detail.

\section{Concentration or mass of the contaminant}

The passage of hazardous substances through the skin is governed by a diffusion process that is driven by the concentration of the contaminant on the skin (29). It has been suggested, therefore, that the concentration of the contaminant on the skin, rather than the mass, determines the internal exposure, and, therefore, the assessment of this quantity should provide a more reliable indicator of exposure than mass (30). Although the mass of the contaminant may not be directly important for dermal uptake, it is a measure of the reservoir of contamination on the skin that is potentially available for dermal uptake. The distinction between the concentration and mass of the contaminant on the skin may, therefore, be trivial when the concentration of the material handled is constant (eg, single source with constant concentration) or when there is not much material (ie, mass) available for uptake. However, in many occupational settings, these requirements will not be met, and, in these circumstances, it can be argued that the metric that should be assessed is the actual concentration of the contaminant on the skin, rather than the mass of the contaminant on the skin.

However, current monitoring techniques only measure mass (eg, skin loading). Therefore, an estimate of the concentration of the contaminant on the skin can only be assessed subjectively at present, using the concentration of the contaminant in the source or on contaminated surfaces as an estimate of the concentration of the hazardous substance on the skin. Information about the concentration of the contaminant in the source can be retrieved historically, at least in certain occupational settings, as was shown in two epidemiologic studies $(21,25)$. Retrospective exposure assessment studies should therefore attempt to collect as much information as possible about the concentration of the active ingredient in the source(s) or on contact surface(s) over time, together with information about the actual mass of the contaminant on the skin.

\section{Surface area exposed}

The surface area exposed, together with the concentration or mass of the contaminant, determines the total amount available for uptake at a certain time point. The first consideration in an evaluation of the surface area exposed is the identification of the parts of the body exposed. The identification depends largely on inherent process characteristics (eg, aerosol generation, ejection of particles, and splashes), which describe the interaction between the source, process, and equipment with the work environment and the dermal exposure pathway (eg, deposition, direct contact, immersion), which describes the interaction of the workers' skin with the work environment.

Several studies using visualization techniques have examined the surface area exposed under certain exposure scenarios. These studies were almost exclusively restricted to the agricultural setting and to spray painting activities (31-33), and therefore the possibility to generalize the results are limited. Nonetheless, these studies clearly showed that the distribution of the dermal contaminant is, in most cases (if not all), inhomogeneous across the body. The notion of an inhomogeneous distribution of contaminants across the body was recognized long before it was actually visualized properly (34). To overcome this problem, it became general practice to divide the body into a number of specific body locations to meet the assumption of a homogeneous exposure distribution within the defined body locations $(35,36)$. Although, there is no limit to the number of body locations that one could define, for practical reasons, the body is often divided into nine (35) or ten (36) distinctive regions, namely, the head, trunk (chest and back), upper arms, forearms, hands, legs, thighs, lower legs, and feet. It is assumed that each location is homogeneously exposed and that the area exposed is equivalent to the skin surface area of that particular location. However, Fenske (32) demonstrated, among pesticide applicators, that the actual proportion of the skin surface of specific body regions receiving exposure is relatively small (4-22\%) and highly variable. Estimation of the actual surface area exposed within an a priori defined body location is, therefore, imperative for an accurate estimate of dermal exposure. The estimation is, however, difficult if no visualization data are available or can be collected (because the tracer cannot be added to the source of exposure) or the occurrence of dermal exposure is random like, for instance, a spill. In this case, it may only be possible to derive an estimate of the exposed surface area by carefully considering the involved exposure pathways and work practices. In general, it can be assumed that dermal exposure originating from the immersion or deposition of airborne particulates on the skin will result in a more uniform exposure distribution for a specific body location than exposure originating from splashes and direct skin contact with contaminated surfaces.

The exposed surface area not only depends on external factors, however, but also on subject-specific parameters, including the use of (protective) clothing, individual work practices, and the actual skin surface area of the person in question. The use of protective clothing [the term protective clothing is used here, but 
it also includes gloves and regular clothing, which can provide protection from dermal exposure as well (37$38)]$ is an important determinant of the actual skin area exposed. The most basic evaluation of this parameter is covered and uncovered skin. For the skin covered by protective clothing, the area exposed and mass of the contaminant on the skin depends on the protection factor provided by the clothing. However, little is known about the field effectiveness of protective clothing, which depends both on the selection (eg, type of material, degree of permeation, fit) and use (eg, replacement, wear and tear, cleanliness). A complicating factor is that clothing can potentially increase the uptake of the chemical through the skin by occlusion (39). Occlusion describes the process of increased hydration and temperature of the skin due to the coverage of the contaminated skin by clothing (or any other material), which thereby increases absorption secondarily. In addition, clothing may actually pull contaminants inside the clothing as a result of a "pumping effect" caused by normal body movements during work (40). Furthermore, studies have demonstrated that hands cannot be considered protected from exposure even if "appropriate" gloves are worn. This lack of protection may be due to contamination on the interior surface of the gloves, removal of gloves when fine hand movement is required, and handling of the outside of the gloves when putting them on or taking them off $(37,41)$. It could well be, therefore, that, although clothing can substantially reduce dermal contact with chemicals by reducing both the area exposed and the mass of the dermal contaminant, it may not decrease uptake by as much as one would expect on the basis of a laboratory-evaluated protection factor (42). Conservative estimates of the protection provided by protective clothing should be applied, as proper use or proper functioning often cannot be assumed.

The total skin surface area obviously differs from person to person. These individual differences are seldom taken into consideration, except in a study by vanRooij et al (43). In this study each body location was assigned a fixed percentage, based on the area distribution of the body surface of a "standard man" (36), of the person's total body surface area estimated on the basis of the subject's weight and height (44). Although this approach may seem unnecessarily precise, inclusion of subject-specific estimates for the total body surface area of a person may, in some circumstances, be important because it has been estimated that up to a threefold difference in surface area can be found among adult men (45). However, it is unclear whether differences in a person's total body surface area automatically results in differences in the actual surface area exposed. In some environmental settings this assumption probably holds true (eg, for showering or swimming in contaminated waters). In these situations it seems reasonable to assume a constant relationship between a person's total skin surface area and the exposed surface area. However, for many scenarios within industrial settings, such a direct relationship cannot be assumed, particularly if the surface area of a contaminated tool or product limits the area of contact with, for example, the hands. Extrapolation of dermal exposure from the estimated skin surface area may be necessary only when a homogeneous exposure distribution across the body or within a body location is a justifiable assumption and a direct association between the total individual skin surface and the exposed surface area can be assumed (eg, immersion).

Thus retrospective exposure assessment should focus on identifying likely exposed body locations on the basis of visualization studies or the involved exposure pathways and use of protective clothing. In a second step, the actual skin surface area exposed within exposed body locations needs to be assessed. Incorporating differences in the individual skin surface area seems less important, given the underlying assumptions and limited effect.

\section{Frequency and duration of exposure}

In addition to estimating the concentration or mass of the contaminant on the skin and the surface area of the exposed skin, two components of time (eg, frequency and duration of dermal contact) have to be considered. Depending on the mass transport process involved and the penetration rate of the contaminant, either the frequency or duration of the exposure will be more important. In the case of an event-based exposure process (eg, intermittent exposures or sporadic contact transfer from surface to skin through accidental contact) in combination with a high percutaneous penetration rate of the contaminant (ie, quick absorption), the exposure will be driven by the frequency of contacts with the contaminant (figure 1). In other words, if the percutaneous penetration rate is not rate limiting, the component of time can be described by the frequency of dermal contacts with the contaminant. In contrast, if the mass transport process is continuous (eg, deposition of airborne particulates, condensation of fumes on the skin, solvent vapors) or the uptake of the contaminant through the skin is rate limiting or uptake is limited by the rate of dissolution of the contaminant in the sweat layer (eg, particulates), the time component is best described by the duration of exposure. In this case, it is important to realize that, in contrast to inhalation exposure, dermal exposure does not necessarily stop after the workshift has ended, but continues until the contaminant is removed from the skin either by absorption, evaporation, mechanical loss, or deliberate removal from the skin. Recently, Kissel \& Fenske (46) proposed a model for estimating dermal absorption for re-entry workers. 
The model can easily be applied to other occupations (figure 2). It considers retention of the contaminant on the skin during (i) a net accumulation phase (ie, the workshift) with continuous exposure until a washing event and (ii) a postworkshift period (until the washing event), over which the external load declines as the material is absorbed (neglecting evaporation and mechani-

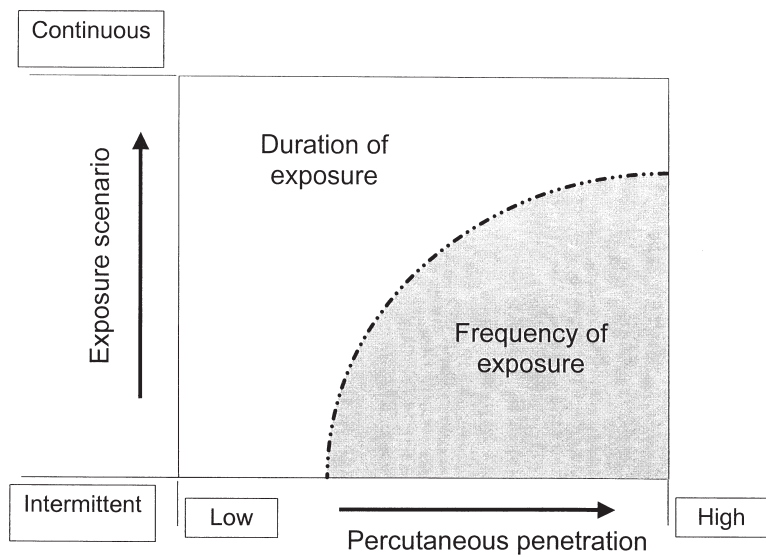

Figure 1. Concept for using frequency or duration as the components of time. The dotted line and consequent gray area indicates the point where the penetration rate of the contaminant is not rate limiting relative to the exposure scenario (ie, intermittent to continuous). cal losses from the skin). This model assumes a $100 \%$ removal efficiency of the contaminant from the skin by a single washing event. However, even if the skin is washed, it may still be contaminated. For example, two studies found pesticide residues on the skin of pesticide workers 1 to 3 days after exposure $(47,48)$. In addition, some reports have shown incomplete removal of PCB from the skin by washing (49) and actual enhancement of dermal uptake for hydrocortisone due to washing of the hands (50). The duration of the postworkshift period should therefore carefully be considered for the exposure of interest and, if necessary, adjusted according to knowledge of removal efficiencies for the particular contaminant.

Furthermore, it should also be recognized that, although hands may be washed directly after the workshift, areas such as the arms and face are less likely to be cleaned immediately. Wearing contaminated clothing beyond the workshift can also prolong dermal exposure, as it serves as a continuous reservoir of contaminant and may continue to be a source even after the clothing has been laundered (51). The fact that dermal exposure (and consequently dermal uptake) can continue through residual contamination on the skin or on clothing after the actual activity or work has stopped is unique to dermal exposure and should be carefully evaluated in the assessment of dermal exposure.

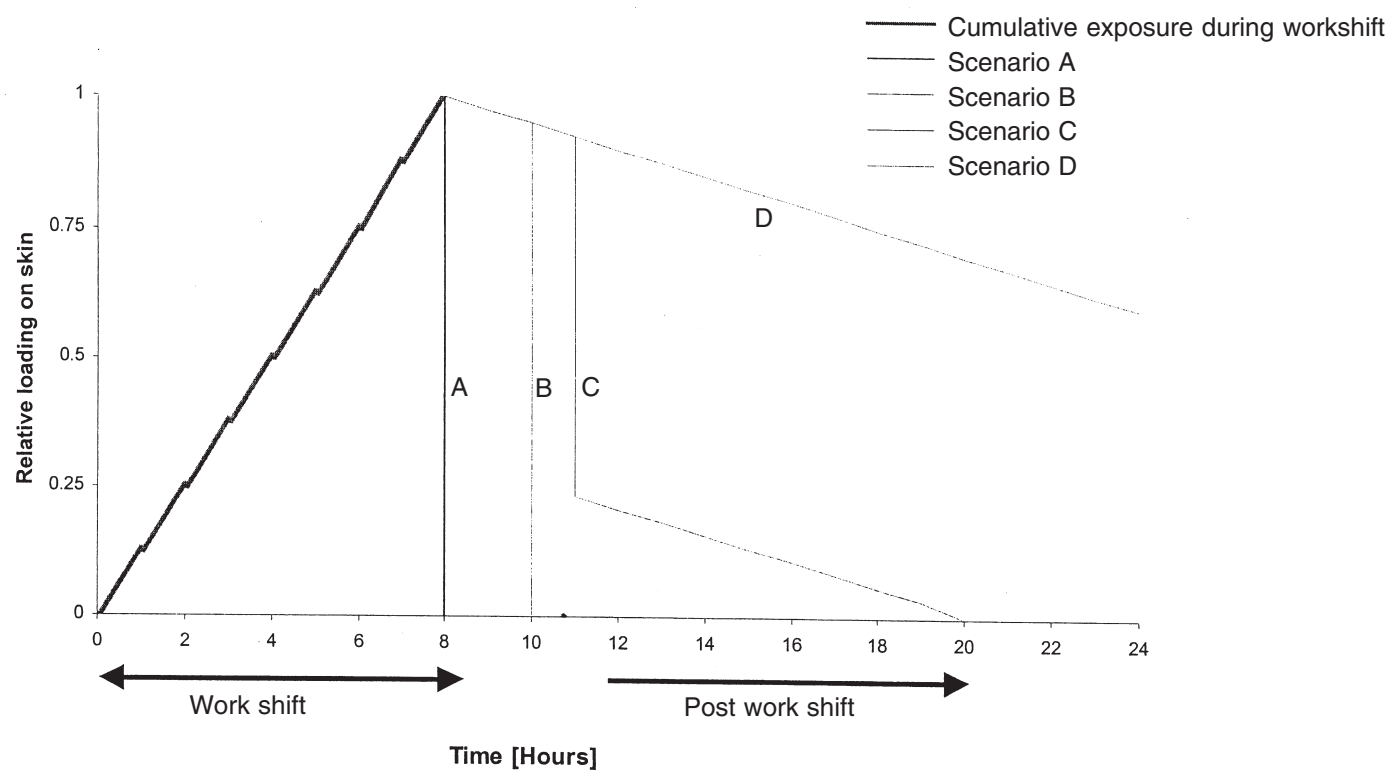

Figure 2. Illustrative exposure scenarios with explicit treatment of the time dependence of absorption after the workshift. Exposure is accumulated over the workshift (shown as 8 hours). Scenario A: effective washing event immediately after finishing work; scenario B: effective washing event some time after the workshift (shown here as 2 hours); scenario C: partial removal of contaminants ( $75 \%$ effective) by washing event some time after the workshift (shown here as 3 hours); scenario D: no washing event after the workshift. For the last three scenarios (B-D) the chemical remaining on the skin at the end of the shift or after partial decontamination of the skin continues to be absorbed. [Figure modified based on an example developed by Kissel \& Fenske (46)] 
For retrospective dermal exposure assessment, it is thus important to collect information about the duration and frequency of exposure through careful consideration of work practices. After the percutaneous penetration rate is considered, an evaluation can be made of whether duration or frequency of exposure is the best component of time. In addition, information on skin and clothing decontamination procedures can be used to estimate the contribution of postworkshift exposure.

\section{Variability issues}

\section{Exposure}

Recognition of the personal, temporal, and spatial variability in exposure concentrations is imperative for the design of efficient and effective sampling strategies, for the interpretation of existing exposure data, and for assigning exposure estimates in an epidemiologic survey (52). The distribution of exposure over the body (ie, spatial variability) has been studied extensively, at least in the relative sense. In contrast, a formal evaluation of temporal and personal variability in dermal exposure measurements has been almost entirely absent. Recently, a database of dermal exposure measurements (DERMDAT) was constructed to analyze these two types of dermal exposure variability (14). These analyses showed median values of the total and within- and between-worker geometric standard deviations of 2.55, 1.98 and 1.47 , respectively, which are strikingly similar to those published for respiratory exposure (53).

From this database, several factors were identified that increased the between-worker variance, for example, working in an indoor environment, localized sources, and a random measurement strategy (14). Interestingly, these factors only influenced the between-worker variance and not the day-to-day variance. That none of the studied factors explained the day-to-day variance suggests that dermal exposure is event-based in many occupational settings, with a certain probability that a contaminated surface is touched by a worker or a splash or spill lands on the skin. Additional justification for the event-based scenario of dermal exposure is that the sampling method partly determined the temporal exposure variability of the hand (14). Patches, which measured exposure at only a small part of the hand or wrist, exhibited more day-to-day variability than the hand wash method, which measured the total hand and wrist exposure. This finding suggests that the occurrence of dermal exposure at a specific spot on the body depends, at least partly, on chance.

The third variance component for dermal exposure is the between-body location component (ie, among skin locations, spatial variability). This source of variability is generally considered to be the most prominent component. Interestingly, in the DERMDAT database, the day-to-day variance component almost disappeared when the variability of the between-body location was taken into account (14). This finding suggests that the day-to-day variability in dermal exposure is important for a specific body location, but not for the overall average of total body exposure. A valid assessment of total dermal exposure thus depends on an accurate assessment of both the exposure distribution across the body and the intensity of the exposure at different body locations. Dermal exposure estimates based on measurements on a single day or a single body location should, therefore, be interpreted with caution.

In epidemiology, it is often assumed, for practical reasons, that workers employed in the same job at a given location are uniformly exposed. This assumption has led to observational schemes for classifying workers into homogeneous exposure groups on the basis of job title, location, and other identifiable features of the work environment (54). In figure 3 the cumulative distribution of the between- and within-worker values of the $R_{0.95}$ 's [the ratio of the 97.5 th and 2.5 th percentiles of the log-normal distribution of the between $\left({ }_{\mathrm{b}} R_{0.95}\right)$ and within $\left({ }_{\mathrm{w}} R_{0.95}\right)$ worker distribution], respectively, are shown for both inhalation and dermal exposure, using the WAUNC (53) and the DERMDAT (14) databases. The exposure groups were defined by job title and factory (eg, homogeneously exposed groups) and, in case of dermal exposure, also by body location. It can be seen that, in general, the range in the day-to-day variability exceeds the range in the between-worker variability for both inhalation and dermal exposure. According to Rappaport (55) an observational group is uniformly exposed if ${ }_{\mathrm{b}} R_{0.95}$ is less than two, while the Health and Safety Executive uses a criterion for uniformity that translates to ${ }_{\mathrm{b}} R_{0.95}$ being less than four (56). For inhalation exposure, $25 \%$ of the groups had $95 \%$ of the individual mean exposures within a factor of two, and $50 \%$ had $\mathrm{a}_{\mathrm{b}} R_{0.95}$ of less than four. For dermal exposure $40 \%$ of the groups had a ${ }_{\mathrm{b}} R_{0.95}$ within a factor of two, and $49 \%$ had a ${ }_{\mathrm{b}} R_{0.95}$ of less than four. Although the DERMDAT database has very limited power for generalization, it can be deducted that considerable variation in dermal exposure levels among persons within "homogeneous exposure groups" (as defined by job title and body location) exists. Thus, as is the case for inhalation exposure, exposure assessors should not rely blindly on observational schemes to guarantee that groups of workers are uniformly exposed by either dermal contact or by inhalation. Retrospective exposure assessment strategies should therefore adopt methods to address the variance components, if possible, by carrying out pilot studies to estimate the contribution from these sources of variance 
Dermal exposure

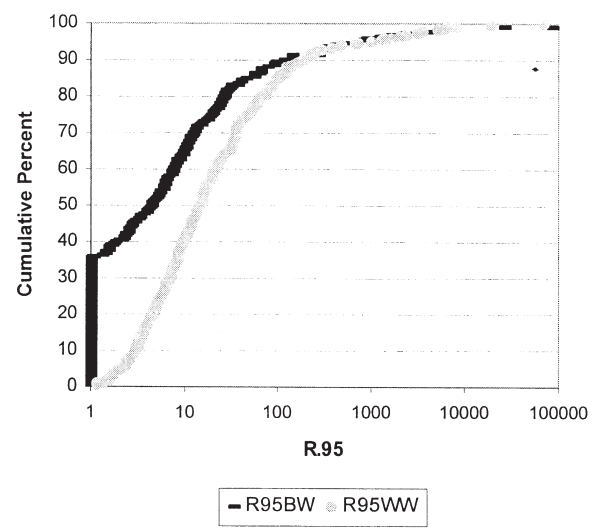

Inhalation exposure

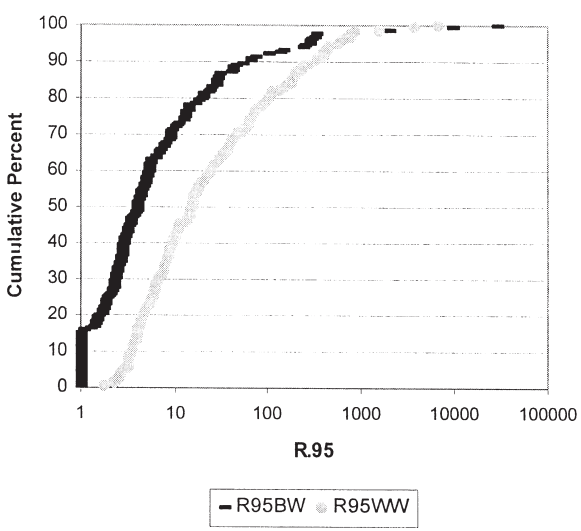

Figure 3. Cumulative distribution of the between worker (R95BW) and within worker (R95WW) ratio of the 97.5th and 2.5th percentiles of the lognormal distribution for dermal (283 groups of workers based on job title, factory and body location) and inhalation (165 groups of workers based on job title and factory) exposure. [Figures adapted from Kromhout et al (53) and Kromhout \& Vermeulen (14)]

and identifying their determinants to optimize exposure assessment methods, strategies, and grouping schemes. These pilot studies should perform dermal exposure measurements at various body locations of several persons on multiple days.

\section{Uptake}

Dermal absorption depends not only on the time-dependent concentration of the contaminant on the skin surface, but also on compound-specific factors (eg, polarity, chemical structure, volatility), the presence of absorption enhancing concomitant exposures, and skinspecific factors. Compound-specific factors are inherent to the chemical under consideration and therefore are unlikely to lead to differences in percutaneous penetration between workers or groups of workers. On the other hand, concomitant exposures to ethanol and other short-chain alkanols, polyethylene glycols, acetone, and other solvents have been found to enhance the percutaneous penetration of several compounds $(40,57)$ and could result in substantial differences in uptake between (groups of) workers.

Skin-specific factors include the condition of the skin, skin temperature, skin thickness, skin perfusion, and lipid-protein makeup. The latter three differ substantially by anatomic region and thus affect dermal absorption rates. The effect of the anatomic region on percutaneous absorption has revealed reasonably similar results for a variety of chemicals, the head, neck, and scrotum being indicated as the more permeable body areas (58). To determine what parts of the body are most important in occupational exposure assessment, we calculated mean relative absorption rates per body location relative to the forearm by taking the ratio between the percutaneous penetration rate of each body location to that of the forearm (table 1). This value, multiplied by the actual surface area, results in a permeability-corrected skin surface area, which indicates the importance of each location as a function of the surface area and the percutaneous penetration rate. The scrotal area was not included in the calculation because it is not a standard measurement site in occupational dermal exposure assessment (65). The corrected surface areas indicate the importance of the upper body region (eg, the head, neck, and upper arms), which accounts for more than $40 \%$ of the permeability-corrected surface area. It should be noted that the estimated average percutaneous absorption coefficients are based on a very limited dataset and that differences in the percutaneous absorption rates among anatomic regions cannot be assumed to be independent of the physicochemical properties of the tested chemicals (eg, mostly lipophilic compounds) (38). Although the results of these studies seem reasonably consistent in their relative ranking of the different body locations, the exact estimates are imprecise. Nevertheless, they could be used when no better data are available. This finding suggests that, if a limited number of body locations is to be measured, they should be selected on the basis of the anticipated exposure distribution, taking into account the permeability of the specific body locations. For many industrial settings, the basic set of dermal measurements should therefore include the hands, head, neck, and shoulders.

Dermal uptake can be increased by several factors, of which occlusion and adverse skin conditions are the most important (66). The effect of occlusion has been shown to increase in vivo percutaneous absorption for several pesticides by a median factor of 3.5 (range 2.39.2) (67). Occlusion could therefore change substantially the absorption rates of the various body locations covered by clothing or gloves (39). This effect could well 
Table 1. Mean relative percutaneous penetration rates and permeability-corrected surface areas by body region. $(A M=$ arithmetic mean)

\begin{tabular}{|c|c|c|c|c|c|c|c|c|}
\hline \multirow{2}{*}{$\begin{array}{l}\text { Body } \\
\text { location }\end{array}$} & \multirow[t]{2}{*}{$\mathrm{Na}^{\mathrm{a}}$} & \multirow[t]{2}{*}{ Compounds ${ }^{b}$} & \multicolumn{3}{|c|}{ Percutaneous penetration ratios ${ }^{c}$} & \multirow{2}{*}{$\begin{array}{l}\text { Surface area } \\
\quad\left(\mathrm{cm}^{2}\right)\end{array}$} & \multirow{2}{*}{$\begin{array}{l}\text { Percentage of total } \\
\text { surface aread }\end{array}$} & \multirow{2}{*}{$\begin{array}{l}\text { Permeability-corrected } \\
\text { percentage of total } \\
\text { surface area }^{\mathrm{e}}\end{array}$} \\
\hline & & & $\mathrm{AM}$ & SD & Range & & & \\
\hline Head & 8 & a to $\mathrm{g}$ & 2.55 & 1.34 & $0.95-4.75$ & 1300 & 6.2 & 11.8 \\
\hline Neck & 2 & a, c & 8.45 & 6.43 & $3.9-13.0$ & 260 & 1.2 & 7.8 \\
\hline Forearms & 11 & a to $\mathrm{k}$ & 1.00 & . & . & 1210 & 5.8 & 4.3 \\
\hline Hands & 3 & $a, b, c$ & 1.37 & 0.55 & $0.8-1.9$ & 820 & 3.9 & 4.0 \\
\hline Upper arm & 1 & k & 2.04 & . & . & 2910 & 14.0 & 21.1 \\
\hline Trunk & 10 & a to j & 1.22 & 0.47 & $0.62-2.10$ & 6840 & 32.8 & 29.7 \\
\hline Upper leg & 3 & $h, i, k$ & 1.00 & 0.23 & $0.75-1.21$ & 3820 & 18.3 & 13.6 \\
\hline Lower leg & 2 & $\mathrm{c}, \mathrm{k}$ & 0.48 & 0.08 & $0.42-0.53$ & 2380 & 11.4 & 4.0 \\
\hline Feet & 5 & $a, b, c, h, i$ & 0.83 & 0.56 & $0.14-1.60$ & 1310 & 6.3 & 3.9 \\
\hline Scrotum & 2 & $\mathrm{a}, \mathrm{c}$ & 26.90 & 21.4 & $11.8-42.0$ & $\cdot$ & $\cdot$ & $\cdot$ \\
\hline Total & . & . & . & . & . & 20850 & 100 & 100 \\
\hline
\end{tabular}

a Number of compounds for which percutaneous penetration data were available for the particular body location (58, 60-64).

b Chemicals studied: (a) parathion, (b) malathion, (c) hydrocortisone, (d) benzoic acid sodium, (e) caffeine, (f) benzoic acid, ( $g$ ) acetyl salicyl acid, (h) fentanyl, (i) sufentanil, (j) ketoprofen, (k) polycyclic aromatic hydrocarbons.

c The percutaneous penetration ratio of the particular body location relative to that of the forearm calculated for each study and then averaged per body location.

${ }^{\mathrm{d}}$ Adapted from reference 59.

${ }^{e}$ The permeability-corrected percentage of the total surface area was calculated by multiplying the surface area $\left(\mathrm{cm}^{2}\right)$ of a particular body location by the mean percutaneous absorption ratio for that location divided by the total permeability-corrected surface area $\left(\mathrm{cm}^{2}\right)$. The total permeability-corrected surface area was calculated as the sum of all individual permeability-corrected body locations and equaled $28181 \mathrm{~cm}^{2}$. For example, head: $[(1300 \times 2.55) / 28181] \times 100=11.8$.

counterbalance most of the differences in percutaneous absorption rates between the different body areas indicated in table 1. However, in general, it is difficult to take occlusion into account due to the limited knowledge on the occurrence of occlusion and its actual effect on percutaneous absorption under field circumstances.

Adverse skin conditions (eg, hydration, dryness, skin diseases) have been shown to affect percutaneous absorption under both laboratory and field circumstances (67-71). Of the adverse skin conditions, skin abrasions (eg, dermatitis, cuts, burns) have been shown to have a potentially dramatic effect on percutaneous absorption. The median effect of skin abrasions on in vivo percutaneous absorption for several pesticides was estimated to be 6.3 (range 2.6-9.5) (67). However, for some exposures, like paraquat, this effect could even be more dramatic, with almost $0 \%$ absorption through intact skin but $100 \%$ absorption through damaged skin (72). It seems, therefore, essential to take the presence of adverse skin conditions into account when systemic uptake through the skin is considered, especially since skin diseases are estimated to account for $9-35 \%$ of all occupational diseases (73-74).

In prospective and case-referent studies, questions should be asked about the occurrence of adverse skin conditions (ie, dermatitis, cuts). However, several studies have shown that standard questionnaires for identifying skin diseases or symptoms have limited use in epidemiologic studies because the overall validity is moderate with generally a moderate-to-high specificity (range 71-99\%) but lower sensitivity (range 31-76\%) (75-80). Recently, questionnaires using pictures of visual symptoms of skin diseases have been developed, and it is anticipated that their validity, and thus their applicability for epidemiologic research, will improve. In retrospective cohort studies, one could rely on medical records to assess skin diseases. However, relatively few people with skin complaints seek medical assistance (81), and, therefore, this approach seems futile. In circumstances in which no direct information on skin disorders can be obtained, the overall risk for the occurrence of skin diseases in a particular occupational group, if known based on the peer review literature, could be used as an exposure modifier at the group level. However, because the magnitude of the effect seems to be related to the physicochemical properties of the substance and because the relationship between exposure and adverse skin conditions (linear, nonlinear) is unknown, it would be preferable to include the occurrence of adverse skin conditions as an interaction term with the dermal exposure estimates in epidemiologic analyses.

\section{Applicability to exposure assessment for epidemiologic studies}

\section{Variability issues}

Proper assignment of dermal exposure estimates in epidemiologic studies requires knowledge about the level 
of exposure (eg, intensity, exposed surface areas, duration), exposure variability (personal, temporal, spatial), and variability in uptake. The importance of accounting for exposure variability in inhalation exposures is widely recognized, and methods to account for these sources of variability in epidemiologic exposure assessment strategies have been well described in the literature $(54,82-85)$. Approaches have been developed to address the variability between groups, the variability among people within groups, and the variability over time. Careful consideration of these sources of variability increases the efficiency and sensitivity of the assessment and reduces bias.

These approaches also can be used in dermal exposure assessments, and many of the conclusions for inhalation assessment apply to dermal exposure assessment. The between-body location variance is, however, unique to dermal exposure, and methodologies have not yet been established to incorporate this source of variability into the design of exposure assessment strategies. Nevertheless, in principle, the between-body location variance can be considered a component of the day-today variance. When the spatial variability component is low, relatively few body areas would have to be measured to arrive at a good estimate of total body exposure.

In a study by Vermeulen et al (86) the relation between the dermal exposure of individual skin regions and total body contamination was investigated among rubber workers. A strong correlation (Pearson correlation $r=0.87$ and $r=0.95$ for production workers and technical engineers, respectively) was found between exposure at the wrist and total body exposure; this finding suggests that, for this particular occupational setting and epidemiologic purposes, dermal exposure could be estimated solely from the exposure of the hands. This assumption allowed the investigators to reduce the number of body locations that needed to be measured to estimate dermal exposure levels and facilitated the collection of repeated measurements from more persons to address personal and temporal variability issues. Careful consideration of the minimum number of measurements necessary to arrive at an accurate estimate of total body exposure, based on spatial autocorrelation analyses, could significantly increase the effectiveness and efficiency of the exposure assessment strategy.

Variability in the percutaneous penetration rates among body areas is generally small, only the head, neck, and scrotal areas having substantially different absorption rates than other locations. When these body locations are likely to be significantly exposed, the differences in their absorption rates should be taken into account. An example of such a situation can be found in a study by de Cock et al (87), who studied fruit growers applying pesticides. In this study an association was found between tetrahydrophthalimide in urine and captan exposure on the neck, but not with the total dermal exposure. In exposure situations in which only one area predominates exclusively or no significant exposure occurs to the head, neck, or scrotal area, it seems reasonable to assume a single percutaneous absorption rate for all body areas, as is common in models used for the dermal uptake of environmental contaminants (8890) or merely the use of the external dermal exposure level as the estimate of exposure.

\section{Exposure assessment}

Methodologies that can be used to estimate dermal exposure levels depend strongly on the available exposure data. If comprehensive measurement data are available, (retrospective) exposures can be estimated using stochastic modeling of the dermal measurement data. Unfortunately, historical measurements are scant, except perhaps for pesticides, for which over 100 studies have been published that provide quantitative exposure data. It has to be noted, however, that probably many more dermal measurement data exist that have never been published in peer-reviewed scientific journals. However, even with the over 100 pesticide studies, historical assessment of the pesticide data is problematic due to the range of pesticides measured, the use of different measurement techniques (patches versus whole body), the measurement of different body locations, exposure measurements outside (potential exposure) and inside (actual exposure) clothing, and differences in extrapolation procedures. In addition, these studies often lack a comprehensive evaluation of the determinants of dermal exposure, and this lack limits their value in assessments of historical dermal exposure levels. Another source of information that could be used to develop pesticide exposure scores is the Pesticide Handlers Exposure Database (PHED) (91) and the European Predictive Operator Exposure Model (EUROPOEM) (92). These pesticide exposure databases reflect exposures to operators (mixers or loaders and applicators) under "representative" field circumstances, by which is meant highly standardized use scenarios. There is, therefore, some concern about its relevance to actual exposure situations because of the controlled, almost experimental, conditions under which the application occurs (eg, best case monitoring). However, relative comparisons between different application methods and various types of protective equipment can be inferred from these databases.

Statistical modeling of current dermal exposure measurements to identify and quantify exposure determinants may be a more fruitful approach. Although issues concerning differences in measurement techniques and strategies apply as well, "successful" examples of this approach can be found in the literature. These studies applied a single measurement method and collected 
auxiliary data, on the basis of which determinants of dermal exposure were identified $(86,93-97)$ and historical exposure trends were inferred (98). Such studies could render estimates of exposure determinants under realistic field scenarios and provide estimates of the field effectiveness of protective clothing.

When few or no direct dermal measurements are available, process-specific empirical models can be used to assess dermal exposure. An example of such a process-specific model has been described by Brouwer et al (99) for spray painting. Factors incorporated in the model were spray technique, the object being sprayed, the workers' individual work practices, and deposition based on a conceptual-empirical algorithm. Dosemeci et al (100) developed an algorithm to estimate the intensity of applicators' pesticide exposure. Weighting factors for the task of mixing, the application method, equipment repair status, and personal protective use were estimated using the published literature and professional judgment. Preliminary validation data of the spray painting and applicators' model showed reasonable-to-good rank correlations with the measured exposure ( $\mathrm{r}=0.82$ and $\mathrm{r}=0.95$, respectively) $(99,100)$. However, both validation studies used "semi-experimental" settings that focused on a single specific task. Therefore, correlation between these models and "real-life" full-shift exposure scenarios will probably be lower. It will be important to see if these process-specific models are able to explain between-worker differences or only the temporal variance (task-to-task observation). Additional validation of these models is therefore necessary to evaluate their usefulness in epidemiologic studies. Nevertheless, the use of process-specific models to estimate dermal exposure might hold some promise for dermal exposure assessment and will, as a minimum, be helpful in providing key factors that need to be addressed in the estimation of dermal exposure in these particular exposure scenarios.

A generic dermal exposure model has been developed by the Health and Safety Executive in the United Kingdom (101) for risk assessment purposes. This model (eg, estimation and assessment of substance exposure) does not allow for individual work practices, and it ranks workplaces in broad bands of exposure, assuming therefore, homogeneous exposures within the workplace. In addition, the model deals only with skin exposure of the hands and forearms. Preliminary validation data of the model for estimating and assessing substance exposure has shown that the model overestimates exposure at the hand and forearms by orders of one to two magnitudes (102). Given the limitations of the model, its applicability in dermal exposure assessment in epidemiologic studies seems, at least for the time being, limited.

Subjectively assessing exposures through the use of expert judgment relies heavily on the familiarity of the expert with the studied exposure situation, which may be an inherent problem for dermal exposure assessment as the current level of knowledge is generally poor. Because expert judgment requires, in principal, the least amount of a priori measurement data or qualitative exposure information, it can be used the most readily in epidemiologic research. This ease of use also explains the initial popularity of expert judgment in the beginning of inhalation exposure assessment for epidemiologic purposes. In expert evaluation, dermal exposure levels can be estimated (from measurement data), semiquantitative estimates can be developed from knowledge of the workplace or a relative ranking of job titles or individuals may be developed. In a study by Lansink et al (103), an expert panel ranked airless spray painters from three workplaces according to their dermal exposure levels. The inter-rater differences were so high that no consensus in the rating of painters could be achieved (104). A high inter-rater difference was also found in a study of fruit growers exposed to pesticides (105). Nevertheless, pesticide experts and occupational hygienists were able to rank daily exposure levels during pesticide spraying in a meaningful way $(\mathrm{r}=0.64)$. The validity of subjective exposure assessment by experts likely depends on the exposure scenario, the available qualitative information and measurement data, and the level of expertise of the raters, as is needed for inhalation exposure (106). In any case, expert panels should not be too small (three to five) and mathematical (eg, summary estimates, axiomatic models, or Bayesian models) or behavioral (eg, consensus) approaches should be used to combine the expert opinions, as differences within expert groups can be considerable (107). One approach to minimize this variability would be to develop a formal structure for evaluating dermal exposures based on a priori rigorous evaluation criteria.

\section{Concluding remarks}

The assessment of dermal exposure is still in its infancy, and many problems have been discussed with regard to the measurement and estimation of dermal exposure. The inclusion of quantitative dermal exposure estimates in retrospective cohorts will therefore be very limited for many years to come. However, this limitation should not automatically lead to the conclusion that the dermal route should not be included in current studies, because potentially severe misclassification could occur by the omission of a significant exposure route (108). For example, in the case of pesticides, 90-95\% of the absorbed dose has been found to be from dermal exposure (34). The extent and the structure of the misclassification will depend greatly on the relation between inhalation and 
Table 2 Overview of considerations for the estimation of (historical) dermal exposure levels in occupational epidemiologic research.

\begin{tabular}{|c|c|c|}
\hline Parameters to be considered & Relative importance a & Current level of knowledge b \\
\hline \multicolumn{3}{|l|}{ Intensity of the contaminant } \\
\hline Concentration of contaminant on skin & ++++ & - \\
\hline Mass of contaminant on skin & +++ & + \\
\hline Concentration of contaminant in the source or on a surface & ++ & $+/++$ \\
\hline Exposure determinants to develop process-specific algorithms & ++ & + \\
\hline \multicolumn{3}{|l|}{ Location and surface area exposed } \\
\hline Identification of exposed areas by visualization techniques or multiple sample sites & ++++ & +/++ \\
\hline Observation of exposure pathways (immersion, airborne deposition, direct contact) & +++ & t/++ \\
\hline Observation of process characteristics (aerosol generation, ejection of particles, splashes) & +++ & t/++ \\
\hline Determinants of exposure distribution and surface area exposed (eg, clothing) & +++ & + \\
\hline Individual's total body surface & + & +++ \\
\hline \multicolumn{3}{|l|}{ Duration and frequency of exposure } \\
\hline Frequency and duration of exposure & ++++ & $-/+$ \\
\hline Skin decontamination behavior or procedures & ++++ & $-/+$ \\
\hline Clothing decontamination or procedures & $+/++$ & $-/+$ \\
\hline \multicolumn{3}{|l|}{ Exposure variability } \\
\hline Personal, temporal and spatial variability & ++++ & $-/+$ \\
\hline Uniform exposure groups & +++ & $-/+$ \\
\hline \multicolumn{3}{|l|}{ Uptake modifiers } \\
\hline Adverse skin conditions & +++ & - \\
\hline Occlusion (clothing, work climate) & +/++ & - \\
\hline Body location, especially head, neck and scrotal area & ++ & $-/+$ \\
\hline
\end{tabular}

a $+=$ mildly, $++=$ moderately, $+++=$ very, $++++=$ extremely important.

b $-=$ poor; $+=$ limited; $++=$ fair; $+++=$ good.

dermal exposure estimates and the way the exposure estimates are used (continuous, categorical). Several investigations have studied the correlation between dermal and airborne exposure estimates and found correlation coefficients ranging from as low as 0.06 to as high as 0.99 , with a median correlation coefficient of $r=0.4$ $(37,43,57,86,94,107,109,110)$. As these statistics indicate, assuming that the optimal grouping strategy for inhalation and dermal exposure is the same is often not justified, because exposure sources and pathways for the two exposure routes may have distinct characteristics.

We have given an overview of the methods applied in epidemiologic studies, described the basic parameters to be considered when dermal exposure is assessed, and described procedures that can be applied in current epidemiologic research. The relative importance of these parameters has been indicated in table 2. It goes without saying that the importance of these parameters depends heavily on the specific scenario under consideration, but the presented ratings can be used as a starting point in the exposure assessment procedure. In addition, we have indicated the current level of knowledge about these parameters. Overall, it can be anticipated that, in the coming years, retrospective dermal exposure assessment for epidemiologic research will generally be based on expert judgment and, to some degree, on process-specific exposure models. It should be stressed that, to increase the comparability across studies that rely on expert judgment, an attempt should be made to document the derivation of quantitative dermal exposures, even if only basic and limited information is available.

Field studies collecting quantitative dermal exposure data and statistical modeling to identify exposure determinants will, however, be imperative for progress in this field. In addition, the identification of the structure and magnitude of exposure variability (personal, temporal, and spatial) will provide information for more efficient and more effective measurement and grouping strategies. Some degree of standardization may be beneficial for current measurement methods, and it would increase comparability between studies. We urge that special attention be given to studies comparing different measurement methods, identifying dermal exposure determinants, evaluating exposure variability, and developing grouping strategies.

\section{Acknowledgments}

The authors thank Berna van Wendel de Joode for her valuable comments on an earlier draft of the manuscript.

\section{References}

1. Mulhausen JR, Damiano J. A strategy for assessing and managing occupational exposures. Fairfax (VA): American 
Industrial Hygiene Association, 1998.

2. Fenske RA. Dermal exposure: a decade of real progress. Ann Occup Hyg 2000;44:489-491.

3. Kauppinen TP. Assessment of exposure in occupational epidemiology. Scand J Work Environ Health 1994;20 special issue:19-29.

4. Armstrong BG. Effect of measurement error on epidemiological studies of environmental and occupational exposures. Occup Environ Med 1998;55:651-656.

5. Thomas D, Stram D, Dwyer J. Exposure measurement error: influence on exposure-disease. Relationships and methods of correction. Annu Rev Public Health 1993;14:69-93.

6. Checkoway H, Rice $\mathrm{CH}$. Time-weighted averages, peaks, and other indices of exposure in occupational epidemiology. Am J Ind Med 1992;21:25-33.

7. Brouwer DH, Boeniger MF, van Hemmen J. Hand wash and manual skin wipes . Ann Occup Hyg 2000;44:501-10.

8. Cherrie JW, Brouwer DH, Roff M, Vermeulen R, Kromhout $\mathrm{H}$. Use of qualitative and quantitative fluorescence techniques to assess dermal exposure. Ann Occup Hyg 2000;44: 519-22.

9. Soutar A, Semple S, Aitken RJ, Robertson A. Use of patches and whole body sampling for the assessment of dermal exposure. Ann Occup Hyg 2000;44:511-8.

10. Axelson O. Some recent developments in occupational epidemiology. Scand J Work Environ Health 1994;20 special issue:9-18.

11. Rice C, Harris RL Jr, Lumsden JC, Symons MJ. Reconstruction of silica exposure in the North Carolina dusty trades. Am Ind Hyg Assoc J 1984;45:689-96.

12. Seixas NS, Robins TG, Becker M. A novel approach to the characterization of cumulative exposure for the study of chronic occupational disease. Am J Epidemiol 1993;137: 463-71.

13. Burstyn I, Kromhout H, Kauppinen T, Heikkila P, Boffetta P. Statistical modelling of the determinants of historical exposure to bitumen and polycyclic aromatic hydrocarbons among paving workers. Ann Occup Hyg 2000;44:43-56.

14. Kromhout H, Vermeulen R. Temporal, personal and spatial variability in dermal exposure. Ann Occup Hyg 2001;45: 257-73.

15. Chen SY, Zhang ZW, He FS, Yao PP, Wu YQ, Sun JX, et al. An epidemiological study on occupational acute pyrethroid poisoning in cotton farmers. Br J Ind Med 1991;48:77-81.

16. Kishi M, Hirschhorn N, Djajadisastra M, Satterlee LN, Strowman S, Dilts R. Relationship of pesticide spraying to signs and symptoms in Indonesian farmers. Scand J Work Environ Health 1995;21:124-33.

17. Ory FG, Rahman FU, Katagade V, Shukla A, Burdorf A. Respiratory disorders, skin complaints, and low-back trouble among tannery workers in Kanpur, India. Am Ind Hyg Assoc J 1997;58:740-6.

18. Ory FG, Rahman FU, Katagade V, Shukla A, Burdorf A. Assessment of exposure to chemical agents and ergonomic stressors in tanneries in Kanpur, India. Am Ind Hyg Assoc J 1997;58:732-9.

19. Driscoll T, Mandryk J, Corvalan C, Nurminen M, Hull B, Rogers A, et al. Health status and exposure of workers at a pilot brown coal liquefaction plant in Australia, 1985-1991. Occup Med 1995;45:239-46.

20. Chase KH, Wong O, Thomas D, Berney BW, Simon RK. Clinical and metabolic abnormalities associated with occupational exposure to polychlorinated biphenyls (PCBs). J Occup Med 1982;24:109-14.
21. Stewart PA, Zaebst D, Zey JN, Herrick R, Dosemeci M, Hornung R, et al. Exposure assessment for a study of workers exposed to acrylonitrile. Scand J Work Environ Health 1998;24 Suppl 2:42-53.

22. Blair A, Stewart PA, Zaebst DD, Pottern L, Zey JN, Bloom $\mathrm{TF}$, et al. Mortality of industrial workers exposed to acrylonitrile. Scand J Work Environ Health 1998;24 suppl 2:25-41.

23. Brouwer DH, Brouwer EJ, van Hemmen JJ. Estimation of long-term exposure to pesticides. Am J Ind Med 1994;25: 573-88.

24. Verberk MM, Brouwer DH, Brouwer EJ, Bruyzeel DP, Emmen HH, Van Hemmen JJ, et al. Health effects of pesticides in the flower-bulb culture in Holland. Med Lav 1990;81:530 41.

25. Paustenbach DJ, Price PS, Ollison W, Blank C, Jernigan JD, Bass RD, et al. Reevaluation of benzene exposure for the Pliofilm (rubberworker) cohort (1936-1976). J Toxicol Environ Health 1992;36:177-231.

26. Crump KS. Risk of benzene-induced leukemia: a sensitivity analysis of the pliofilm cohort with additional follow-up and new exposure estimates. J Toxicol Environ Health 1994; 42:219-42.

27. Paxton MB, Chinchilli VM, Brett SM, Rodricks JV. Leukemia risk associated with benzene exposure in the pliofilm cohort. II. Risk estimates. Risk Anal 1994;14:155-61.

28. Schneider T, Vermeulen R, Brouwer DH, Cherrie JW, Kromhout H, Fogh CL. Conceptual model for assessment of dermal exposure. Occup Environ Med 1999;56:765-73.

29. Fiserova BV. Relevance of occupational skin exposure. Ann Occup Hyg 1993;37:673-85.

30. Cherrie JW, Robertson A. Biologically relevant assessment of dermal exposure. Ann Occup Hyg 1995;39:387-92.

31. Brouwer DH, Lansink CM, Cherrie JW, van Hemmen JJ. Assessment of dermal exposure during airless spray painting using a quantitative visualisation technique. Ann Occup Hyg 2000;44:543-9.

32. Fenske RA. Nonuniform dermal deposition patterns during occupational exposure to pesticides. Arch Environ Contam Toxicol 1990;19:332-7.

33. Roff MW. Dermal exposure of amateur or non-occupational users to wood-preservative fluids applied by brushing outdoors. Ann Occup Hyg 1997;41:297-311.

34. Durham WF, Wolff T. Measurement of the exposure of workers to pesticides. Bull World Health Organ 1962;26:75-91.

35. Environmental Protection Agency (EPA). Development of statistical distribution or ranges of standard factors used in exposure assessments. Washington (DC): EPA, 1985. EPA, 600/8-85/010.

36. Popendorf WJ, Leffingwell JT. Regulating OP pesticide residues for farmworker protection. Residue Rev 1982;82:125201.

37. Aprea C, Sciarra G, Lunghini L, Centi L, Ceccarelli F. Evaluation of respiratory and cutaneous doses and urinary excretion of alkylphosphates by workers in greenhouses treated with omethoate, fenitrothion, and tolclofos-methyl. Am Ind Hyg Assoc J 2001;62:87-95.

38. Stewart PA, Fears T, Nicholson HF, Kross BC, Ogilvie LK, Zahm SH, et al. Exposure received from application of animal insecticides. Am Ind Hyg Assoc J 1999;*:208-12.

39. Qiao GL, Chang SK, Riviere JE. Effects of anatomical site and occlusion on the percutaneous absorption and residue pattern of 2,6-[ring-14C]parathion in vivo in pigs. Toxicol Appl Pharmacol 1993;122:131-8.

40. Ceschel GC, Maffei P, Lombardi BS. Correlation between 
the transdermal permeation of ketoprofen and its solubility in mixtures of a $\mathrm{pH} 6.5$ phosphate buffer and various solvents. Drug Deliv 2002;9:39-45.

41. Maddy KT, Wang RG, Winter CK. Dermal exposure monitoring of mixers, loaders and applicators of pesticides in California. Sacramento (CA): California Department of Food and Agriculture, 1983. HS-1069.

42. Brouwer DH, Marquart H, Van Hemmen JJ. Proposal for an approach with default values for the protection offered by PPE, under European new or existing substance regulations. Ann Occup Hyg 2001;45:543-53.

43. van Rooij JG, Bodelier BM, de Looff AJ, Dijkmans AP, Jongeneelen FJ. Dermal exposure to polycyclic aromatic hydrocarbons among primary aluminum workers. Med Lav 1992;83:519-29.

44. Environmental Protection Agency (EPA). Interim guidance for dermal exposure assessment. Washington (DC): EPA, 1991. EPA/600/8-91/011A.

45. Phillips LJ, Fares RJ, Schweer LG. Distributions of total skin surface area to body weight ratios for use in dermal exposure assessments. J Expo Anal Environ Epidemiol 1993;3:331-8.

46. Kissel J, Fenske R. Improved estimation of dermal pesticide dose to agricultural workers upon reentry. Appl Occup Environ Hyg 2000;15:284-90.

47. Wolfe H, Durham WF, Batchelor G. Health hazards of some dinitro compounds. Arch Environ Health 1961;3:468-75.

48. Ringenberg V. Industrial hygiene characterisation of pesticide exposure of Hamilton county park district workers. Cincinnati $(\mathrm{OH})$ : National Institute for Occupational Safety and Health, 1988. HHE no 87-241.

49. Wester RC, Bucks DA, Maibach HI, Anderson J. Polychlorinated biphenyls (PCBs): dermal absorption, systemic elimination, and dermal wash efficiency. J Toxicol Environ Health 1983;12:511-9.

50. Wester RC, Noonan PK, Maibach HI. Frequency of application on percutaneous absorption of hydrocortisone. Arch Dermatol 1977;113:620-2.

51. Clifford NJ, Nies AS. Organophosphate poisoning from wearing a laundered uniform previously contaminated with parathion. J Am Med Assoc 1989;262:3035-36.

52. Kromhout H. Design of measurement strategies for workplace exposures. Occup Environ Med 2002;59:349-54.

53. Kromhout H, Symanski E, Rappaport SM. A comprehensive evaluation of within- and between-worker components of occupational exposure to chemical agents. Ann Occup Hyg 1993;37:253-70

54. Rappaport SM, Kromhout H, Symanski E. Variation of exposure between workers in homogeneous exposure groups. Am Ind Hyg Assoc J 1993;54:654-62.

55. Rappaport SM. Assessment of long-term exposures to toxic substances in air [see comments]. Ann Occup Hyg 1991;35: 61-121.

56. HSE. Monitoring strategies for toxic substances. London: Her Majesty's Stationery Office, 1989. Guidance note EH42.

57. Sartorelli P, Andersen HR, Angerer J, Corish J, Drexler H, Goen T, et al. Percutaneous penetration studies for risk assessment. Environ Toxicol Pharmacol 2000;8:133-52.

58. Feldmann RJ, Maibach HI. Regional variation in percutaneous penetration of $14 \mathrm{C}$ cortisol in man. J Invest Dermatol 1967;48:181-3.

59. Environmental Protection Agency (EPA). Pesticide assessment guidelines: subdivision U. Washington (DC): EPA 1987.

60. Maibach HI, Feldman RJ, Milby TH, Serat WF. Regional variation in percutaneous penetration in man. Pesticides. Arch Environ Health 1971;23:208-11.

61. Rougier A, Lotte C, Maibach HI. In vivo percutaneous penetration of some organic compounds related to anatomic site in humans: predictive assessment by the stripping method. J Pharm Sci 1987;76:451-4.

62. Roy SD, Flynn GL. Transdermal delivery of narcotic analgesics: $\mathrm{pH}$, anatomical, and subject influences on cutaneous permeability of fentanyl and sufentanil. Pharm Res 1990;7: $842-7$.

63. Shah AK, Wei G, Lanman RC, Bhargava VO, Weir SJ. Percutaneous absorption of ketoprofen from different anatomical sites in man. Pharm Res 1996;13:168-72.

64. van Rooij JG, de Roos JH, Bodelier-Bade MM, Jongeneelen FJ. Absorption of polycyclic aromatic hydrocarbons through human skin: differences between anatomical sites and individuals. J Toxicol Environ Health 1993;38:355-68.

65. Organisation for Economic Co-operation and Development (OECD). Guidance document for the conduct of occupational exposure to pesticides during agricultural application. Paris: OECD, 1997.

66. Wester RC, Maibach HI. In vivo percutaneous absorption and decontamination of pesticides in humans. J Toxicol Environ Health 1985;16:25-37.

67. Maibach H, Feldmann RJ. Systemic absorption of pesticides through the skin of man. Washington (DC): US Government Printing Office, 1974:120-7.

68. McBride JS, Altman DG, Klein M, White W. Green tobacco sickness. Tob Control 1998;7:294-8.

69. Vermeulen R, Bos RP, Pertijs J, Kromhout H. Exposure related mutagens in urine of rubber workers associated with inhalable particulate and dermal exposure. Occup Environ Med. In press.

70. Lumens ME, Ulenbelt P, Geron HM, Herber RF. Hygienic behaviour in chromium plating industries. Int Arch Occup Environ Health 1993;64:509-14.

71. Riihimaki V, Laine A, Savolainen K, Sippel H. Acute solvent-ethanol interactions with special reference to xylene. Scand J Work Environ Health 1982;8:77-9.

72. Hart TB. Paraquat: a review of safety in agricultural and horticultural use. Hum Toxicol 1987;6:13-8.

73. Smit HA, Burdorf A, Coenraads PJ. Prevalence of hand dermatitis in different occupations. Int J Epidemiol 1993;22: 288-93.

74. Vermeulen R, Kromhout H, Bruynzeel DP, de Boer EM, Brunekreef B. Dermal exposure, handwashing, and hand dermatitis in the rubber manufacturing industry. Epidemiology 2001;12:350-4.

75. Berg M, Axelson O. Evaluation of a questionnaire for facial skin complaints related to work at visual display units. Contact Dermatitis 1990;22:71-7.

76. McCurdy SA, Wiggins P, Schenker MB, Munn S, Shaieb AM, Weinbaum Z, et al. Assessing dermatitis in epidemiologic studies: occupational skin disease among California grape and tomato harvesters. Am J Ind Med 1989;16:14757.

77. Susitaival P, Husman L, Hollmen A, Horsmanheimo M. Dermatoses determined in a population of farmers in a questionnaire-based clinical study including methodology validation. Scand J Work Environ Health 1995;21:30-5.

78. Vermeulen R, Kromhout H, Bruynzeel DP, de Boer EM. Ascertainment of hand dermatitis using a symptom-based questionnaire: applicability in an industrial population. Contact Dermatitis 2000;42:202-6. 
79. Meding B, Barregard L. Validity of self-reports of hand eczema. Contact Dermatitis 2001;45:99-103.

80. Yngveson M, Svensson A, Isacsson A. Evaluation of a selfreported questionnaire on hand dermatosis in secondary school children. Acta Derm Venereol 1997;77:455-7.

81. Smit HA, Coenraads PJ, Lavrijsen AP, Nater JP. Evaluation of a self-administered questionnaire on hand dermatitis. Contact Dermatitis 1992;26:11-6.

82. Heederik D, Attfield M. Characterization of dust exposure for the study of chronic occupational lung disease: a comparison of different exposure assessment strategies. Am J Epidemiol 2000;151:982-90.

83. Kromhout H, Heederik D. Occupational epidemiology in the rubber industry: implications of exposure variability. Am J Ind Med 1995;27:171-85.

84. Seixas NS, Sheppard L. Maximizing accuracy and precision using individual and grouped exposure assessments. Scand J Work Environ Health 1996;22:94-101.

85. Tielemans E, Kupper LL, Kromhout H, Heederik D, Houba R. Individual-based and group-based occupational exposure assessment: some equations to evaluate different strategies. Ann Occup Hyg 1998;42:115-9.

86. Vermeulen R, Heideman J, Bos RP, Kromhout H. Identification of dermal exposure pathways in the rubber manufacturing industry. Ann Occup Hyg 2000;44:533-41.

87. de Cock J, Heederik D, Hoek F, Boleij J, Kromhout H. Urinary excretion of tetrahydrophtalimide in fruit growers with dermal exposure to captan. Am J Ind Med 1995;28:24556.

88. McKone TE. Household exposure models. Toxicol Lett 1989;49:321-39.

89. Rao HV, Brown DR. A physiologically based pharmacokinetic assessment of tetrachloroethylene in groundwater for a bathing and showering determination. Risk Anal 1993;13:3749.

90. Roy A, Weisel CP, Lioy PJ, Georgopoulos PG. A distributed parameter physiologically-based pharmacokinetic model for dermal and inhalation exposure to volatile organic compounds. Risk Anal 1996;16:147-60.

91. Environmental Protection Agency (EPA). PHED: pesticide handlers' exposure database version 1.1. Washington (DC): EPA, Health and Welfare Canada, National Agricultural Chemicals Association, 1992. EPA/600/8-91/011A.

92. van Hemmen JJ. EUROPOEM, a predictive occupational exposure database for registration purposes of pesticides. Appl Occup Environ Hyg 2001;16:246-50.

93. Riley DM, Small MJ, Fischhoff B. Modeling methylene chloride exposure-reduction options for home paint-stripper users. J Expo Anal Environ Epidemiol 2000;10:240-50.

94. van Wendel de Joode BN, De Graaf IAM, Wesseling C, Kromhout H. Paraquat exposure of knapsack spray operators on banana plantations in Costa Rica. Int J Occup Environ Health 1996;2:294-304.

95. Kromhout H, Swuste P, Boleij JS. Empirical modelling of chemical exposure in the rubber-manufacturing industry. Ann Occup Hyg 1994;38:3-22.

96. de Cock J, Heederik D, Kromhout H, Boleij JS, Hoek F, Wegh H, et al. Determinants of exposure to captan in fruit growing. Am Ind Hyg Assoc J 1998;59:166-72.
97. Ohayo-Mitoko GJ, Kromhout H, Karumba PN, Boleij JS. Identification of determinants of pesticide exposure among Kenyan agricultural workers using empirical modelling. Ann Occup Hyg 1999;43:519-25.

98. Vermeulen R, de Hartog J, Swuste P, Kromhout H. Trends in exposure to inhalable particulate and dermal contamination in the rubber manufacturing industry: effectiveness of control measures implemented over a nine-year period. Ann Occup Hyg 2000;44:343-54.

99. Brouwer DH, Semple S, Marquart J, Cherrie JW. A dermal model for spray painters, part I: subjective exposure modelling of spray paint deposition. Ann Occup Hyg 2001;45:1523 .

100. Dosemeci M, Alavanja MC, Rowland AS, Mage D, Zahm $\mathrm{SH}$, Rothman N, et al. A quantitative approach for estimating exposure to pesticides in the Agricultural Health Study. Ann Occup Hyg 2002;46:245-60.

101. European Chemicals Bureau. Technical guidance document in support of the commission directive 93/67/EEC on risk assessment for new notified substances and the commission regulation (EC) 1488/94 on risk assessment for existing chemicals. Ispra (Italy): European Chemicals Bureau, 1996.

102. Hughson GW, Cherrie JW. Validation of the EASE expert system for dermal exposure to zinc. In: Marklund S, editor. $\mathrm{X} 2001$ - exposure assessment in epidemiology and practice. Stockholm: National Institute of Working Life, 2001:1719.

103. Lansink CM, van Hengstum C, Brouwer D. Dermal exposure due to airless spraying. Zeist (The Netherlands): TNO Nutrition and Food Research Insititute, 1997. V97.1057.

104. Brouwer D, Lansink CM, Marquart H. Qualitative assessment of dermal exposure: preliminary identification of determinants and an outline of an approach for subjective estimation of exposure. Zeist (The Netherlands): TNO Nutrition and Food Research Institute, 1998. V97.1077.

105. de Cock J, Kromhout H, Heederik D, Burema J. Experts' subjective assessment of pesticide exposure in fruit growing. Scand J Work Environ Health 1996;22:425-32.

106. Kromhout H, Oostendorp Y, Heederik D, Boleij JS. Agreement between qualitative exposure estimates and quantitative exposure measurements. Am J Ind Med 1987;12:551-62.

107. Clemen RT, Winkler RL. Combining probability distributions from experts in risk analysis. Risk Anal 2002;19:187203.

108. Daniell W, Stebbins A, Kalman D, O’Donnell JF, Horstman SW. The contributions to solvent uptake by skin and inhalation exposure. Am Ind Hyg Assoc J 1992;53:124-9.

109. Wolff MS, Herbert R, Marcus M, Rivera M, Landrigan PJ, Andrews LR. Polycyclic aromatic hydrocarbon $(\mathrm{PAH})$ residues on skin in relation to air levels among roofers. Arch Environ Health 1989;44:157-63.

110. Brouwer R, van Maarleveld K, Ravensberg L, Meuling W, de Kort W, van Hemmen JJ. Skin contamination, airborne concentrations, and urinary metabolite excretion of propoxur during harvesting of flowers in greenhouses. Am J Ind Med 1993;24:593-603.

Received for publication: 3 April 2002 